\title{
Correspondence
}

\section{Trial of ketoconazole in allergic bronchopulmonary aspergillosis}

SIR,-I read with interest the report of the trial of ketoconazole in non-invasive pulmonary aspergillosis by $\mathrm{Dr}$ DJ Shale and colleagues (January 1987;42:26-31). It is the first time that an antifungal agent has proved effective in allergic bronchopulmonary aspergillosis.

We were unable to find any benefit from ketoconazole $400 \mathrm{mg} /$ day in an open trial of nine cases of allergic bronchopulmonary aspergillosis. ${ }^{1}$ All the patients relapsed over the next 12 months: three after two months, two after five months, and four between six and 12 months. Relapse was ascertained on biological criteria in all cases (increase of total serum IgE levels, isolation of Aspergillus fumigatus in sputum), associated in five cases with both clinical (increase of asthma or sputum or both) and radiological criteria (pulmonary infiltration or mucoid impaction with atelectasis or both), with clinical criteria alone in three cases, and radiological criteria alone in one case. Another case of allergic bronchopulmonary aspergillosis can be added to this series. This patient presented with psoriasis complicated by erythroderma after treatment with corticosteroids prescribed for the allergic bronchopulmonary aspergillosis. Corticosteroids were withdrawn and, despite treatment with ketoconazole $400 \mathrm{mg} /$ day for eight months, he developed a left upper lobe eosinophilic infiltrate in the second month and mucoid impaction of the right upper lobe bronchus six months later, with an increase of total serum IgE from 1740 to 7842 and $9764 \mathrm{U} / \mathrm{ml}$ on the two occasions. So in our experience ketoconazole was unable to prevent relapse of allergic bronchopulmonary aspergillosis.

Our study was an open trial and we focused on relapse of allergic bronchopulmonary aspergillosis and not on asthma symptom scores. Our patients did not receive inhaled corticosteroids but three received $10-15 \mathrm{mg}$ prednisone, the other patients receiving only sympathominetics and/or theophylline. We are interested to know the follow up of the patients studied by Shale et al after ketoconazole was stopped.

ERIC C FOURNIER

Department de pneumologie Hopital Calmette 59000 Lille, France

1 Fournier EC, Tonnel AV, Wallaert B, Voisin C. Ketoconazole trial in prevention of acute phase of allergic bronchopulmonary aspergillosis [abstract]. Am Rev Respir Dis 1984;129:A32.

** This letter was sent to the authors, who reply below.

SIR,- - The study referred to by Dr Fournier and his colleagues was a small trial similar to our study but open in type and without clear entry criteria. Both studies could be criticised over their small numbers of subjects. Our study was a pilot run for a larger study, which was abandoned because of the reports of liver and other complications associated with treatment with ketoconazole. In such small studies carried out in limited geographical regions and extending over one or two risk periods for exacerbation it is not surprising that differing results may occur.

We cannot answer the question of what happened after cessation of treatment as this was not a part of the initial study. On the basis of this study and the known problems of ketoconazole we would not recommend treatment with this agent in allergic bronchopulmonary aspergillosis.

$$
\begin{array}{r}
\text { DJ SHALE } \\
\text { JH FAUX } \\
\text { DJ LANE } \\
\text { Respiratory Medicine Unit } \\
\text { City Hospital } \\
\text { Nottingham NG5 } 1 P B
\end{array}
$$

\section{Airway response to methacholine during exercise induced refractoriness in asthma}

SIR,-Dr H Magnussen and others (September 1986;41:667-70) reported that refractoriness to exercise induced asthma correlated positively with a reduced response to methacholine. However, from a methodological viewpoint, one should be cautious in interpreting the results and in drawing conclusions from them with respect to the possible role of reduced airway reactivity in the pathogenesis of refractoriness. While the exercise stimulus used to measure refractoriness was applied about 50 minutes after the first exercise test, the methacholine challenge was applied some 40-60 minutes later-that is, 90 minutes after the first exercise test. Refractoriness is not constant over time but is maximum 30-40 minutes after exercise and then decreases gradually over about two hours, ${ }^{1}$ so a different degree of refractoriness might have existed during the methacholine challenge. As measurements of these two indices were not done simultaneously, their comparison appears inappropriate. Moreover, the performance of the second exercise test may itself have altered the patient's degree of refractoriness during the methacholine challenge. ${ }^{2}$

ISRAEL AMIRAV

114A Manachat Street Jerusalem, Israel

1 Edmunds AT, Tooley M, Godfrey $\mathrm{S}$. The refractory period after exercise-induced asthma: its duration and relation to the severity of exercise. Am Rev Respir Dis 1978;117:247-54.

2 Weiler-Ravell D, Godfrey S. Do exercise and antigen induced asthma utilize the same pathway? $J$ Allergy Clin Immunol $1981 ; 67: 391-7$. 